\title{
5-fluorouracil enhances the antitumor effect of sorafenib and sunitinib in a xenograft model of human renal cell carcinoma
}

\author{
MAKITO MIYAKE, SATOSHI ANAI, KIYOHIDE FUJIMOTO, SAYURI OHNISHI, MASAOMI KUWADA, \\ YASUSHI NAKAI, TAKESHI INOUE, ATSUSHI TOMIOKA, NOBUMICHI TANAKA and YOSHIHIKO HIRAO
}

\author{
Department of Urology, Nara Medical University, Nara 634-8522, Japan
}

Received December 10, 2011; Accepted March 13, 2012

DOI: 10.3892/ol.2012.662

\begin{abstract}
Sorafenib and sunitinib are multi-kinase inhibitors with antitumor activity in patients with advanced renal cell carcinoma (RCC). Several studies have evaluated the effect of sorafenib/sunitinib in combination with chemotherapeutic agents in different types of tumor. However, few studies have addressed the activity of fluorinated pyrimidine in combination with sorafenib/sunitinib. In this study, we examined the potential of combination therapy with 5FU and sorafenib/sunitinib in human RCC cell lines. Three human RCC cell lines, ACHN, Caki-1 and Caki-2, were used to assess sensitivity to 5-fluorouracil (5FU), sorafenib and sunitinib alone or in combination using an in vitro cell survival assay. Caki-2 cells demonstrated significantly higher resistance to 5FU and sorafenib as compared to ACHN and Caki-1. Additive antitumor effects of the combination therapy were observed in the in vitro study. There was a tendency for a positive correlation between the sensitivity to sunitinib and platelet-derived growth factor $\beta$ (PDGFR- $\beta$ ) expression levels, which were examined by reverse transcription polymerase chain reaction. Caki-1 xenograft models were prepared by inoculating cells subcutaneously into nude mice, which were divided randomly into six groups: control, 5FU (8 mg/kg/day, intraperitoneally), sorafenib (15 mg/ $\mathrm{kg}$ /day, orally), sunitinib (20 mg/kg/day, orally), and 5FU with sorafenib or sunitinib. The treatments were administered on 5 days each week, and tumor growth was monitored for 42 days following inoculation of cells. Synergistic antitumor effects of the combination therapy were observed in an in vivo study. The resected tumors were evaluated using the $\mathrm{Ki}-67$ labeling index and microvessel density. Both the Ki-67 labeling index and microvessel density were decreased in tumors treated with the combination therapy compared to those treated with sorafenib/ sunitinib alone. These findings suggest that the combination
\end{abstract}

Correspondence to: Dr Makito Miyake, Department of Urology, Nara Medical University, 840 Shijo-cho, Kashihara-shi, Nara 634-8522, Japan

E-mail:mm-ulo@naramed-u.ac.jp

Key words: renal cell carcinoma, 5-fluorouracil, sorafenib, sunitinib, angiogenesis therapy of 5FU with sorafenib/sunitinib may be an effective therapeutic modality for advanced RCC patients.

\section{Introduction}

Treatment options for metastatic renal cell carcinoma (RCC) have been limited because tumors are inherently chemotherapyand radiotherapy-resistant (1). Based on the importance of immune mechanisms in the regulation of tumor growth and progression in metastatic RCC, the therapeutic potential of immunotherapeutic agents such as interleukin-2 (IL2) and interferon- $\alpha$ (IFN $\alpha$ ) has been evaluated. However, only a limited subset of patients benefit from cytokine therapy with objective response rates (ORR) of up to $20 \%$ (2). New treatment strategies for metastatic RCC have therefore been investigated. One of these is to block the signals triggered by angiogenic growth factors, such as vascular endothelial growth factor (VEGF) and platelet-derived growth factor (PDGF) (3). Three angiogenesis-targeting agents have been developed clinically for the management of RCC, namely, the monoclonal antibody bevacizumab (Avastin) and the small-molecule multi-kinase inhibitors (MKIs) sorafenib tosylate (Nexavar) and sunitinib malate (Sutent).

Sorafenib is an oral MKI that inhibits VEGF receptors (VEGFRs) 1-3, PDGF receptor (PDGFR)- $\beta$, and the serine threonine kinase Raf-1, which acts through the mitogenactivated protein kinase/extracellular signal-regulated kinase kinase/extracellular signal-regulated kinase (Raf/MEK/ ERK) signaling pathway and plays a crucial role in cell proliferation and tumorigenesis (4). Sunitinib is an oral MKI against several tyrosine kinase receptors, including VEGFRs 1-3, PDGFR- $\beta$, stem cell factor receptor (KIT), and FMS-like tyrosine kinase-3 (5). Preclinical studies have shown that sorafenib and sunitinib significantly inhibited tumor growth in various carcinomas through the mediation of suppressed angiogenesis and direct antitumor effects (1). Several phase II and III studies of sorafenib and sunitinib revealed that each of these agents is effective as a monotherapy in cytokinerefractory, metastatic RCC $(1,5-7)$. In a pivotal phase III trial of sorafenib, 905 patients were randomly assigned to receive sorafenib (400 mg, orally, twice daily) versus the placebo, and the trial investigators demonstrated the efficacy and safety of sorafenib treatment for advanced RCC (7). A phase III trial evaluating first-line sunitinib $(50 \mathrm{mg}$, orally, once daily for 
4 weeks, followed by 2 weeks without treatment) versus IFN $\alpha$ ( 9 million units 3 times weekly) in 750 patients with metastatic clear cell RCC demonstrated statistically significant improvements in the clinical outcomes with sunitinib compared to those with IFNa (8). However, for further improvement of prognosis, agents with different mechanisms of action such as chemotherapeutic agents are required. In addition to promising preclinical and clinical data culminating in the approval of sorafenib and sunitinib, their mechanism of action, good safety and tolerability indicate that they may be a useful treatment option in combination with conventional chemotherapy for advanced solid cancers. Several studies have evaluated the effect of sorafenib or sunitinib in combination with a variety of anticancer agents in various types of tumor (9-14).

RCC is highly resistant to conventional chemotherapy: vinblastine has been reported to achieve a 6-9\% ORR, and 5-fluorouracil (5FU) achieved a 5-8\% ORR (15). Response rates of immunochemical therapies combining 5FU with IL2 and IFN $\alpha$ ranged from 1.8 to $48 \%(16,17)$. Recently, a phase II multicenter trial involving 45 patients with metastatic RCC revealed the efficacy and safety of S-1, an oral fluorinated pyrimidine that includes tegafur, a prodrug of 5FU (18). These data suggest that $5 \mathrm{FU}$ is likely a good candidate for combination therapy with molecular-targeting agents. However, there are currently few reports addressing the activity of $5 \mathrm{FU}$ in combination with sorafenib and sunitinib.

In this study, we evaluated the therapeutic activity of two MKIs, sorafenib and sunitinib, in combination with 5FU in vitro and in vivo. As $5 \mathrm{FU}$ acts via different mechanisms from those of MKIs, we hypothesized that combination therapy exploiting the antiangiogenic and antiproliferative properties of MKIs along with the cytotoxic properties of 5FU is likely to provide beneficial results.

\section{Materials and methods}

Cell culture. The three established human RCC cell lines, ACHN, Caki-1 and Caki-2, were obtained from the ATCC (Manassas, VA, USA). Cells were maintained in RPMI-1640 growth medium (Nissui, Tokyo, Japan) supplemented with $10 \%$ fetal bovine serum (ICN Biomedicals, Aurora, OH, USA), $100 \mathrm{U} / \mathrm{ml}$ penicillin, and $100 \mu \mathrm{g} / \mathrm{ml}$ streptomycin (Gibco, Grand Island, NY, USA) in a standard humidified incubator at $37^{\circ} \mathrm{C}$ in an atmosphere of $5 \% \mathrm{CO}_{2}$. The study was approved by the ethics committee of Nara Medical Unversity.

Antitumor reagents. 5FU (Sigma-Aldrich, St. Louis, MO, USA) and the 2 MKIs, sorafenib tosylate and sunitinib malate (LC Laboratories, Woburn, MA, USA), were dissolved in dimethyl sulfoxide (DMSO) at a concentration of 50, 200 and $40 \mathrm{mg} / \mathrm{ml}$, respectively. The stock solutions were stored at $-20^{\circ} \mathrm{C}$ prior to use.

Cell viability assay. Cells were seeded in a 96-well plate at a density of 2000 cells/well in growth medium, incubated for $24 \mathrm{~h}$ and treated with the indicated concentrations of 5FU, sorafenib, and sunitinib, alone or in combination. Following incubation of the plates for $72 \mathrm{~h}$, a cell viability assay was performed using the Cell Counting Kit-8 (Dojindo, Kumamoto, Japan) according to the manufacturer's instructions. Sensitivity against antitumor reagents was expressed as the $50 \%$ inhibitory concentration (IC50) as described previously (19). The data were expressed as relative values to untreated cells, which were set to 100 .

RNA extraction and reverse transcription-polymerase chain reaction $(R T-P C R)$. Cells were seeded in 6-well plates at a density of $1 \times 10^{5}$ cells/well in growth medium and incubated for $24 \mathrm{~h}$. Total RNA was extracted using the RNeasy mini kit (Qiagen, Hilden, Germany). Total RNA (1 $\mu \mathrm{g})$ was reverse transcribed in a final volume of $20 \mu \mathrm{l}$ with the High Capacity cDNA Reverse Transcription Kit (Applied Biosystems, Foster City, CA, USA). Primer sequences used in this study and the annealing temperatures are shown in Table I. PCR was performed with cDNA, $0.2 \mu \mathrm{M}$ of each primer, and $10 \mu \mathrm{l}$ of AmpliTaq Gold ${ }^{\circledR}$ PCR Master Mix (Applied Biosystems) under the following conditions: denaturation at $95^{\circ} \mathrm{C}$ for $10 \mathrm{~min} ; 35$ cycles of $96^{\circ} \mathrm{C}$ for $3 \mathrm{sec}$, annealing temperature (as listed in Table I) for $3 \mathrm{sec}$, and $68^{\circ} \mathrm{C}$ for $15 \mathrm{sec}$; and a final extension at $72^{\circ} \mathrm{C}$ for $10 \mathrm{sec}$. PCR products were then electrophoresed in $1.5 \%$ agarose gel and visualized by a transilluminator. Subsequent to verifying the mRNA expression of PDGFR- $\beta$, semi-quantitative RT-PCR for this gene was performed in the three cell lines. The pixel intensity for each band was determined using the ImageJ program (NIH Image, Bethesda, MD, USA) and normalized to the amount of hypoxanthine phosphoribosyltransferase (HPRT).

Immunocytochemical (ICC) staining. Cells were seeded at a density of 50,000 cells/well in a Lab-Tek II 4-well Chamber Slide (Nalge Nunc International, Rochester, NY, USA) and incubated in growth medium for $24 \mathrm{~h}$. To fix the cells, the slides were immersed in $4 \%$ paraformaldehyde solution (Wako, Osaka, Japan) for $20 \mathrm{~min}$ at $4^{\circ} \mathrm{C}$. ICC staining was performed with a streptavidin-biotin complex method using the Histofine SAB-PO kit (Nichirei Co., Tokyo, Japan), according to the manufacturer's instructions. To detect the cell expression of PDGFR- $\beta$, mouse monoclonal anti-PDGFR- $\beta$ (clone 28 ; BD Transduction Laboratories, San Diego, CA, USA) was used as the primary antibody. The specificity of the antibody was assessed by performing a secondary antibody-only control experiment. Slides were counterstained with Meyer's hematoxylin (Muto Chemical, Tokyo, Japan) and mounted with malinol (Muto Chemical).

Treatment in mouse xenograft models. Animal experiments for this study were approved by the institutional animal care and use committee at Nara Medical University. Female athymic BALB/c nu/nu mice ( 8 weeks old) were maintained under pathogen-free conditions and provided with sterile food and water. Caki-1 $\left(2 \times 10^{6}\right)$ in $100 \mu \mathrm{l}$ RPMI-1640 and $100 \mu \mathrm{l}$ of Matrigel (Becton Dickinson, Bedford, MA, USA) were injected subcutaneously into each mouse. Fourteen days following inoculation of the cells, the animals were divided randomly into 6 groups (placebo, 5FU, sorafenib, sunitinib, 5FU plus sorafenib, and 5FU plus sunitinib), and treatment was initiated. MKIs were administered orally once daily using a disposable soft catheter tube (Fuchigami Co., Kyoto, Japan), and 5FU was injected intraperitoneally for 5 consecutive days each week for 4 weeks. Doses of sorafenib, sunitinib and 5FU were 
Table I. Sequence of primers used for reverse transcription-polymerase chain reaction.

\begin{tabular}{|c|c|c|c|}
\hline Primer & Sequence & Annealing temperature $\left({ }^{\circ} \mathrm{C}\right)$ & Fragment size (bp) \\
\hline VEGFR-1 & $\begin{array}{l}\text { 5'-TCATGAATGTTTCCCTGCAA } \\
\text { 5'-GGAGGTATGGTGCTTCCTGA }\end{array}$ & 55 & 123 \\
\hline VEGFR-2 & $\begin{array}{l}\text { 5'-GGTGTTTTGCTGTGGGAAAT } \\
\text { 5'-AAACGTGGGTCTCTGACTGG }\end{array}$ & 60 & 186 \\
\hline VEGFR-3 & $\begin{array}{l}\text { 5'-CCCACGCAGACATCAA } \\
\text { 5'-TGCACAACTCCACGA }\end{array}$ & 50 & 380 \\
\hline PDGFR- $\alpha$ & $\begin{array}{l}\text { 5'-CTCCTGAGAGCATCTTTGAC } \\
\text { 5'-AAGTGGAAGGAACCCCTCGA }\end{array}$ & 50 & 712 \\
\hline PDGFR- $\beta$ & $\begin{array}{l}\text { 5'-AATGTCTCCAGCACCTTCGT } \\
\text { 5'-AGCGGATGTGGTAAGGCATA }\end{array}$ & 58 & 688 \\
\hline HPRT & $\begin{array}{l}\text { 5'-GTTGGATATAAGCCAGACTTTGTTG } \\
\text { 5'-ACTCAACTTGAACTCTCATCTTAGGC }\end{array}$ & 55 & 164 \\
\hline
\end{tabular}

VEGFR, vascular endothelial growth factor receptor; PDGFR, platelet-derived growth factor receptor; HPRT, hypoxanthine phosphoribosyltransferase.

Table II. Antitumor reagent concentration for $50 \%$ cell survival of renal cell carcinoma cell lines.

\begin{tabular}{|c|c|c|c|c|c|c|}
\hline \multirow{2}{*}{$\begin{array}{l}\text { Antitumor } \\
\text { reagent }\end{array}$} & \multicolumn{2}{|c|}{$\mathrm{ACHN}$} & \multicolumn{2}{|c|}{ Caki-1 } & \multicolumn{2}{|c|}{ Caki-2 } \\
\hline & IC50 & $95 \% \mathrm{CI}$ & IC50 & $95 \% \mathrm{CI}$ & IC50 & $95 \% \mathrm{CI}$ \\
\hline $5 \mathrm{FU}(\mu \mathrm{M})$ & 2.28 & $1.99-2.62$ & 1.55 & $1.23-1.96$ & 7.09 & $3.42-14.7$ \\
\hline Sorafenib $(\mu \mathrm{M})$ & 0.76 & $0.46-1.24$ & 0.33 & $0.14-0.81$ & 2.91 & $1.43-5.92$ \\
\hline Sunitinib $(\mu \mathrm{M})$ & 1.95 & $1.39-2.72$ & 2.80 & $1.36-4.52$ & 2.48 & $0.98-8.02$ \\
\hline
\end{tabular}

5FU, 5-fluorouracil; IC50, 50\% inhibitory concentration; 95\% CI, 95\% confidence interval.

15,20 and $8 \mathrm{mg} / \mathrm{kg}$, respectively. Control mice received vehicle alone on the same schedule. Tumor diameters were measured twice per week with electronic calipers, and tumor volumes were calculated using the formula [(width) ${ }^{2} \mathrm{x}$ length] $/ 2\left(\mathrm{~mm}^{3}\right)$. The weight of mice was measured once each week. The mice were sacrificed on day 42, and the tumors were resected and subjected to immunohistochemical (IHC) analysis.

IHC analysis of xenograft tumors. Tumors were fixed in $10 \%$ formaldehyde solution and embedded in paraffin. IHC staining was performed as previously described (18). The primary antibodies and incubation conditions were mouse monoclonal anti-Ki-67 (clone MIB-1, Dako Japan, Kyoto, Japan) in ready-to-use form at room temperature for $30 \mathrm{~min}$ and rabbit polyclonal anti-PECAM1 (CD31) (Santa Cruz Biotechnology, Santa Cruz, CA, USA), 1:500 dilution, $4^{\circ} \mathrm{C}$ overnight. For verification purposes, some tumor cells were counterstained with hematoxylin and eosin.

Microvessel density (MVD) analysis. MVD of xenograft tumors was determined as described previously (20). Briefly, slides that were stained with anti-CD31 antibody were scanned at a low magnification (x40) to identify the highest MVD. The number of stained blood vessels in each of these areas was estimated using a high magnification field (x200). Subsequently, the MVD score was calculated as the mean.

Statistical analysis. PRISM software version 5.00 (GraphPad Software, San Diego, CA, USA) was used for drawing graphs and statistical analyses. $\mathrm{P}<0.05$ was considered to be statistically significant.

\section{Results}

Sensitivity against antitumor reagents in RCC cell lines. The sensitivity of RCC cell lines to the three antitumor reagents, 5FU, sorafenib and sunitinib, was determined by the cell viability assays (Fig. 1A-C), and the sensitivity of the three cell lines was compared by calculating the IC50 and $95 \%$ confidence interval $(95 \% \mathrm{CI})$ of the antitumor reagents (Table II). The IC50 for the treatment with 5FU and sorafenib was significantly higher in Caki-2 cells than that in ACHN 
and Caki-1. The IC50s of sunitinib in ACHN, Caki-1, and Caki-2 were 1.95, 2.80, and 2.48, respectively. Taken together, these results show that Caki-2 cells are more resistant to the antitumor reagents than ACHN and Caki-1 cells.

Expression of receptor tyrosine kinases in RCC cell lines. Sorafenib and sunitinib are capable of inhibiting multiple receptor tyrosine kinases (RTKs) $(4,5)$. RT-PCR, performed to investigate the expression of RTKs in RCC cell lines (Fig. 2A), demonstrated that PDGFR- $\beta$ was expressed in the three cell lines, whereas the expression of VEGFR 1-3 and PDGFR- $\alpha$ was not observed in the cell lines used in our study. Densitometric analysis revealed that the PDGFR- $\beta$ expression levels in ACHN, Caki-1 and Caki-2 cells were 1.0, 0.53 and 0.57 , respectively (Fig. 2A). There was a tendency of a positive correlation between the sensitivity to sunitinib and PDGFR- $\beta$ expression levels ( $\mathrm{p}=0.09$, Pearson's correlation). IHC staining was performed in logarithmically growing cells to verify the protein expression of PDGFR- $\beta$. A strong expression of PDGFR- $\beta$ was detected in the cell membrane of the three RCC cell lines (Fig. 2B). These results suggest that PDGFR- $\beta$ is a key target molecule for predicting the effect of sunitinib in RCC.

Combination of $5 F U$ with MKIs in vitro. To examine whether $5 \mathrm{FU}$ enhanced the effect of MKIs when used in combination, cell viability assays were carried out using various concentrations of antitumor agents (Fig. 3). The treatment concentrations of $5 \mathrm{FU}$ were $0.75,1.5$ and $3.0 \mu \mathrm{M}$. The treatment concentrations of sorafenib and sunitinib were $0.2,1.0$ and $2.0 \mu \mathrm{M}$. In ACHN and Caki-1 cells, MKI treatment combined with 5FU led to additive growth inhibitory effects (Fig. 3, upper and middle panels). The effect of the combination treatment with MKIs and 5FU was lower in Caki-2 cells than in ACHN and Caki-1 cells.

Combination effect of 5FU with MKIs in Caki-1 xenograft tumors. Since our aim was to establish a new therapeutic strategy for advanced metastatic clear cell RCC, we selected the Caki-1 cell line, which is derived from skin metastases of clear cell RCC (21), for the in vivo study. To evaluate the enhanced antitumor effect of MKIs by 5FU in vivo, xenograft mice bearing Caki-1 cells were prepared and treated as described in Materials and methods. The mean tumor volumes $\left(\mathrm{mm}^{3}\right)$ on day 42 following the initial treatment of the 6 groups i.e., placebo, $5 \mathrm{FU}$, sorafenib, sunitinib, $5 \mathrm{FU}$ plus sorafenib, and $5 \mathrm{FU}$ plus sunitinib, were $1284 \pm 218,1175 \pm 155,880 \pm 105$, $799 \pm 105,583 \pm 113$ and $355 \pm 95$, respectively (Fig. $4 \mathrm{~A}, \mathrm{p}=0.03$, sorafenib monotherapy versus sorafenib plus $5 \mathrm{FU} ; \mathrm{p}=0.008$, sunitinib monotherapy versus sunitinib plus $5 \mathrm{FU}$ ). Tumors from mice treated with MKIs were not only smaller, but also paler than the control mice tumors (Fig. 4B). There were no significant differences in the inhibitory effects of sorafenib versus sunitinib monotherapies or sorafenib plus $5 \mathrm{FU}$ versus sunitinib plus 5FU. Yellow skin discoloration was observed in all the mice treated with sunitinib. From treatment initiation until tumor resection, no significant loss of body weight was observed in any of the groups. These findings suggested that the combination of MKIs and 5FU is effective and tolerable as a novel therapeutic modality for metastatic RCC.
A

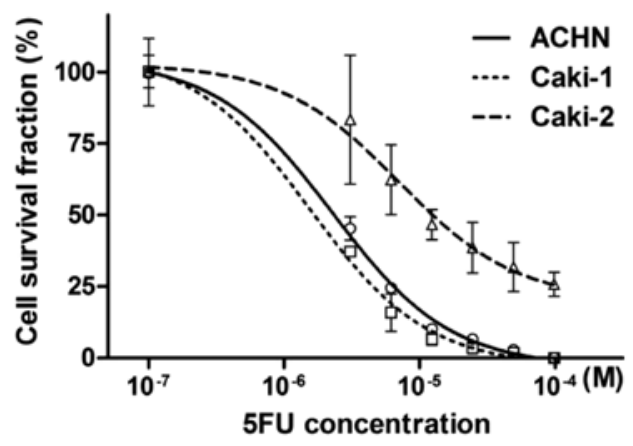

B

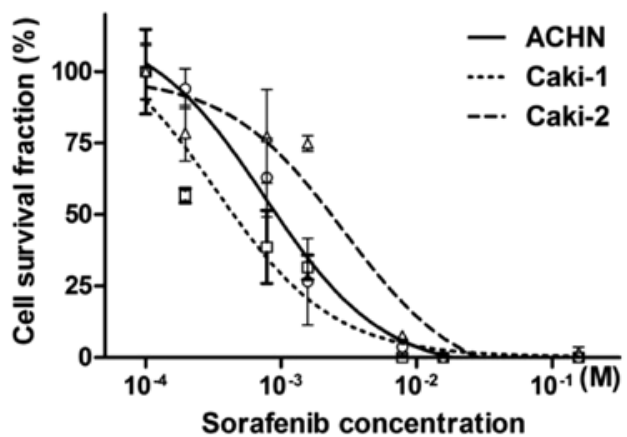

C

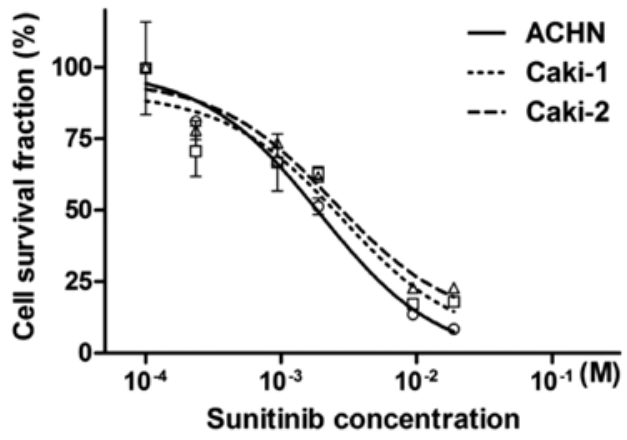

Figure 1 . Growth inhibitory curves of $5 \mathrm{FU}$, sorafenib and sunitinib in vitro. To determine the sensitivity against the three antitumor reagents in the three RCC cell lines, sigmoid curves showing the correlation between the dose and viability were drawn. The cell survival fraction is expressed relative to the untreated cells, set at 100 , and are shown as the mean \pm standard deviation (SD) $(n=4)$. The graphs show results for (A) 5FU, (B) sorafenib and (C) sunitinib

Analyses of resected tumors using the Ki-67 labeling index and $M V D$. To investigate the cell proliferation and angiogenesis status of the treated and control tumors, IHC staining for Ki-67 and CD31 was carried out (Fig. 4C). The Ki-67 labeling index data revealed that tumors from mice treated with MKIs with and without $5 \mathrm{FU}$ had decreased levels of proliferation as compared to the control mice. Combination treatment with sunitinib and $5 \mathrm{FU}$ led to a significant decrease in the Ki-67 labeling index compared to sunitinib monotherapy (Fig. 4D). Assessment of MVD revealed that tumors from mice treated with MKIs with and without 5FU demonstrated decreased MVD compared to the control mice (Fig. 4E). The angiogenesis status was not affected by $5 \mathrm{FU}$ alone or in combination in this in vivo study. The effect of MKI treatment on PDGFR- $\beta$ expression in cancer tissues in vivo was assessed by IHC staining for this receptor, and the results revealed no differences between tissues resected from the 6 treatment groups. 
A

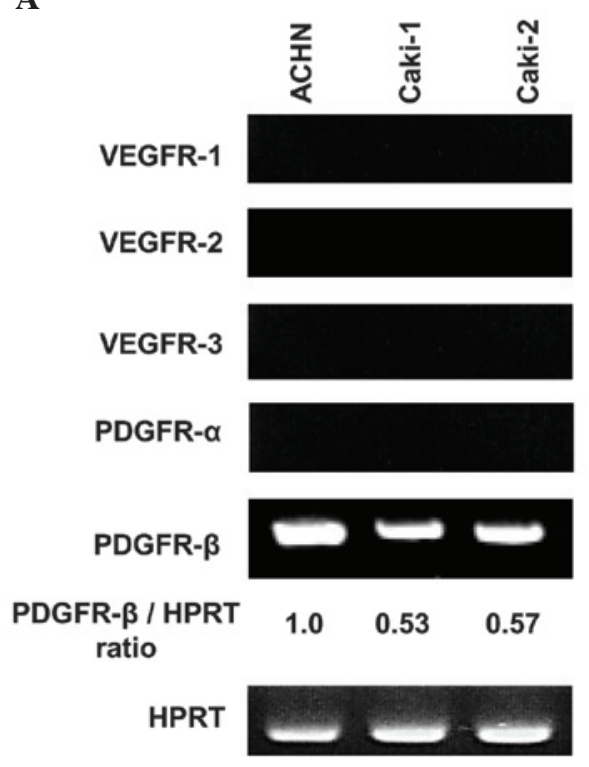

B

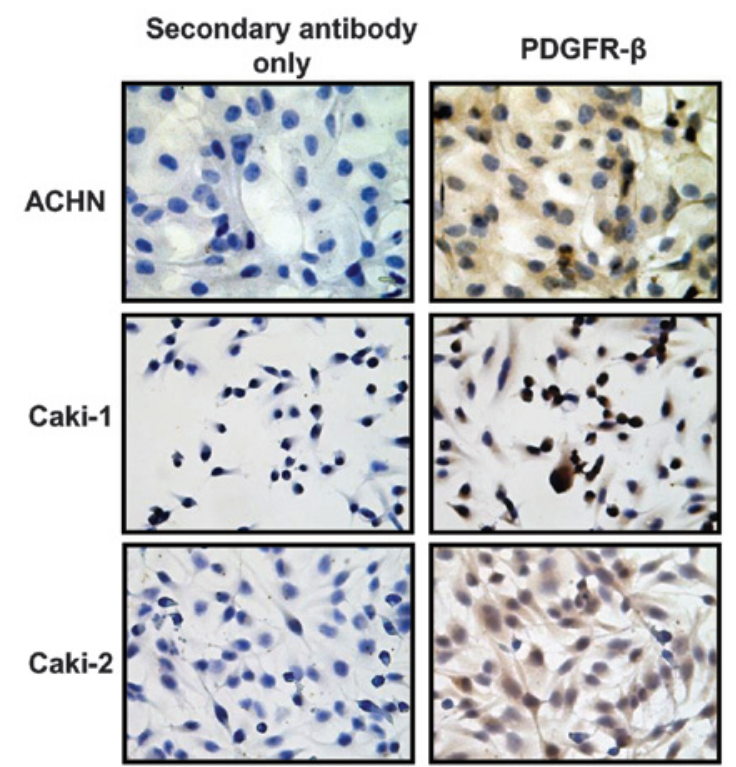

Figure 2. Expression of tyrosine kinase receptors in renal cell carcinoma (RCC) cell lines. (A) Expression levels of 5 tyrosine kinase receptors were examined by reverse transcription-polymerase chain reaction (RT-PCR). A housekeeping gene, hypoxanthine phosphoribosyltransferase (HPRT), was used as an endogenous control. For semi-quantitative RT-PCR, the number of PCR cycles was reduced to 30 cycles. PDGFR- $\beta / H P R T$ expression ratios were calculated based on the densitometric analysis and are expressed as relative values to ACHN, which is set to 1.0. (B) Three RCC cell lines were immunostained in chamber slides to examine the expression of the PDGFR- $\beta$ protein (right row). A secondary antibody-only control experiment was performed (left row). Original magnification, $\mathrm{x} 200$.

A

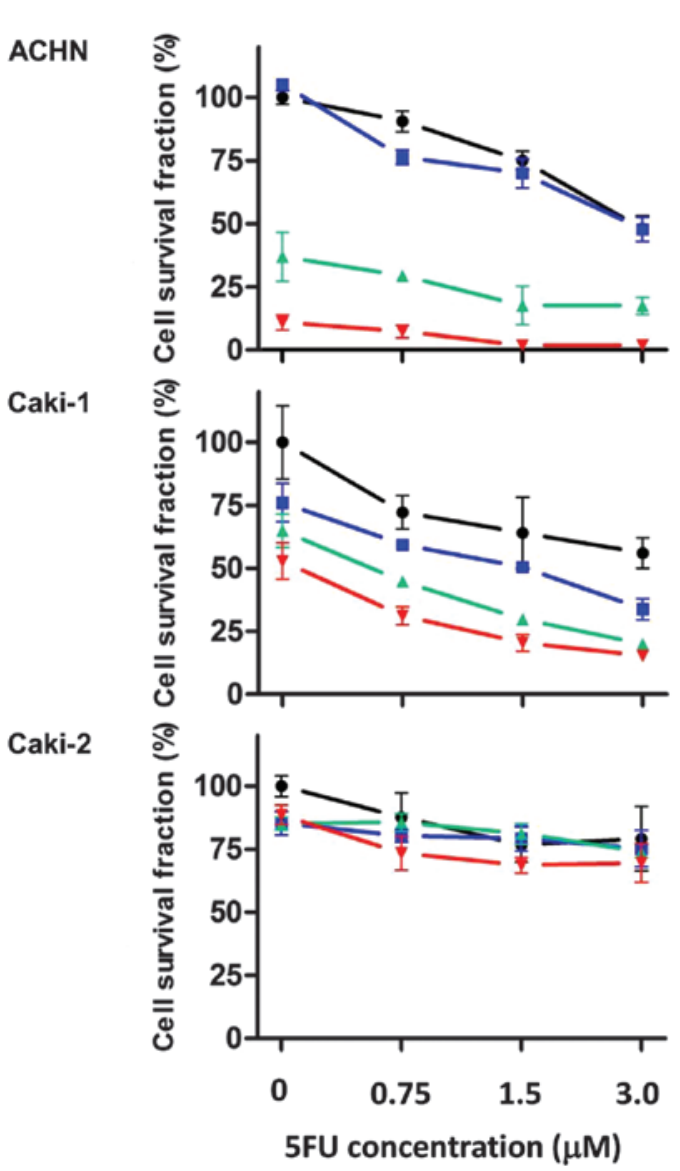

B
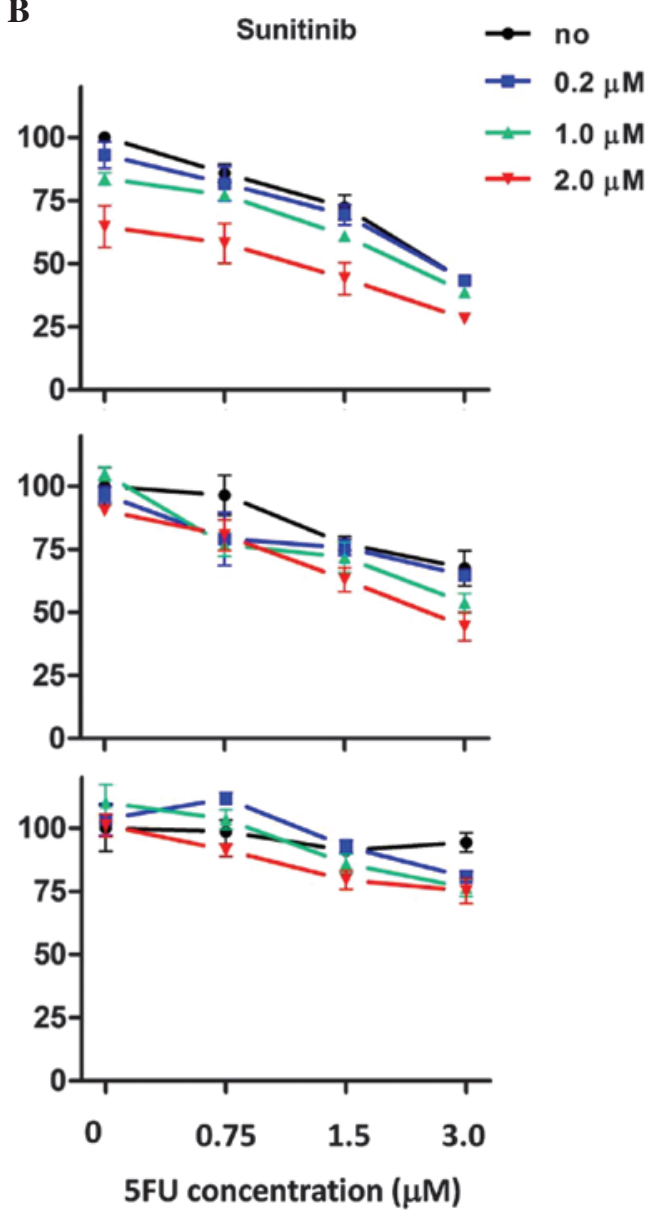

Figure 3. Growth inhibitory effect of the combination of 5-fluorouracil (5FU) with multi-kinase inhibitors in vitro. Three renal cell carcinoma cell lines were treated with escalating doses of $5 \mathrm{FU}(0.75,1.5$ and $3.0 \mu \mathrm{M})$ and (A) sorafenib or (B) sunitinib $(0.2,1.0$ and $2.0 \mu \mathrm{M})$. The cell survival fraction is expressed relative to the untreated cells, set at 100 , and is shown as the mean $\pm S D(n=3)$. 

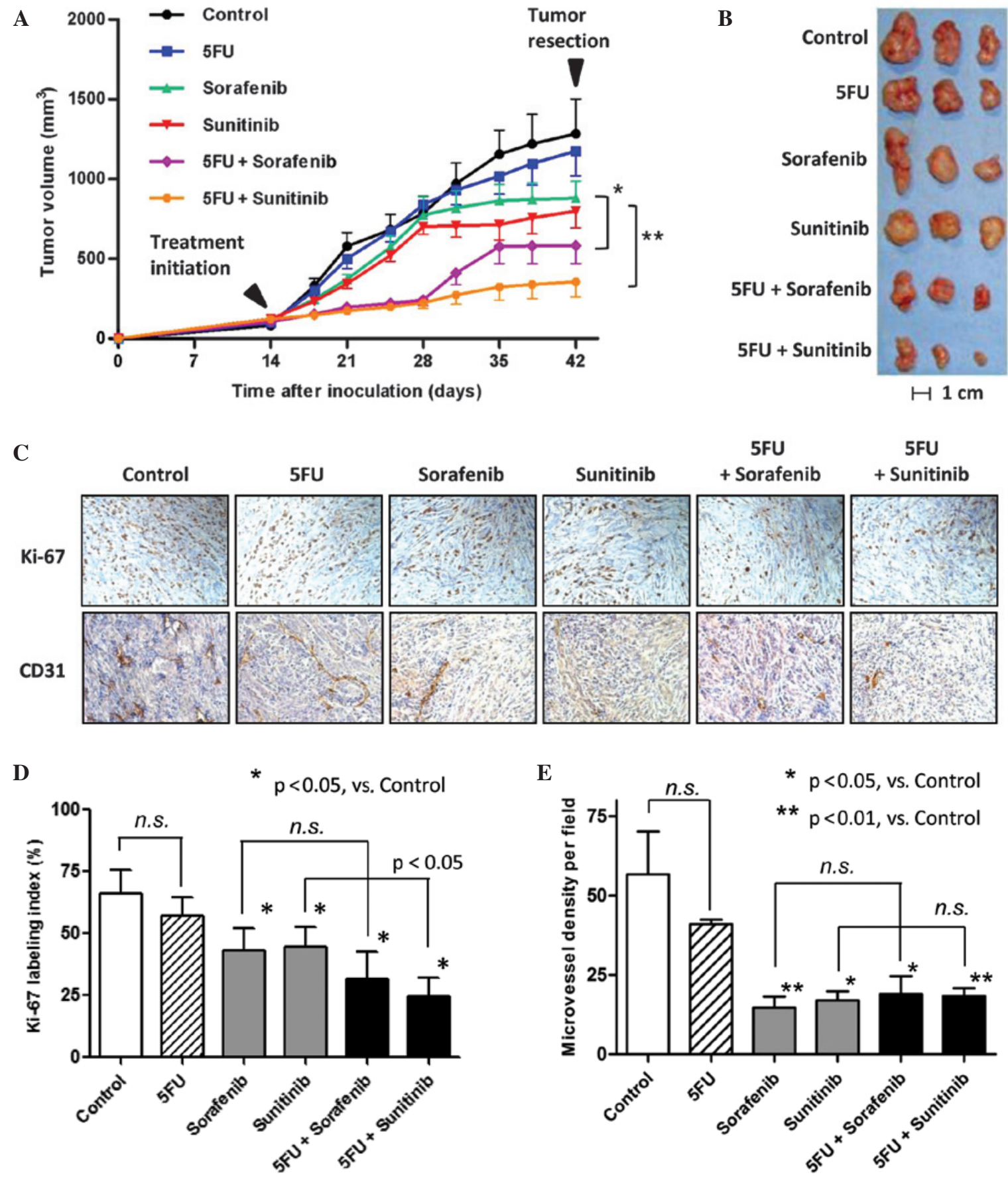

Figure 4. Growth inhibitory effect of the combination of 5-fluorouracil (5FU) with multi-kinase inhibitors in tumor-bearing mice. (A) The tumor volumes of the 6 groups are plotted from day 14 until day 42 after the inoculation of Caki-1 cells. Intraperitoneal administration of $5 \mathrm{FU}(8 \mathrm{mg} / \mathrm{kg} / \mathrm{day})$ and oral administration of sorafenib/sunitinb (15 and $20 \mathrm{mg} / \mathrm{kg} / \mathrm{day}$, respectively) was initiated on day 14 . The data are presented as the mean \pm standard error of the mean (SEM). Statistically significant differences in tumor volumes were observed in response to sorafenib versus $5 \mathrm{FU}$ plus sorafenib ("p $<0.05$ ) and sunitinib versus $5 F U$ plus sunitinib $(* \mathrm{p}<0.01)$. Statistically significant differences were determined by using the Mann-Whitney U test. (B) Imaging of resected tumors. The representative 3 tumors for each treatment group are shown. (C) The resected tumors of the xenograft mice were analyzed by immunohistochemical staining for Ki-67 (upper panels) and CD31 (lower panels). Representative slides of each treatment group are shown. Original magnification, x200. (D) The proliferative activity was evaluated by Ki-67 staining. The percentage of positive tumor cells was then calculated as Ki-67 labeling index using 4 fields. The data are presented as the mean \pm SD. Statistically significant differences were determined using the Mann-Whitney $U$ test. "p $<0.05$. (E) The angiogenesis activity was evaluated by CD31 staining. The data are expressed as the mean $\pm \mathrm{SD} .{ }^{*} \mathrm{p}<0.05,{ }^{* *} \mathrm{p}<0.01$.

\section{Discussion}

Approaches that may maximize the clinical benefit of antitumor therapies should include an evaluation of the appropriate combination regimens, implementation of novel trial designs and the identification of predictive markers of response. The different mechanisms of the antitumor activity of sorafenib/ sunitinib and 5FU and their manageable single-agent safety profiles provide a strong rationale for combining these agents. The findings of the present in vivo study demonstrated that 5FU may enhance the antitumor effect of the two MKIs available in clinical settings (Fig. 4A). 
Several studies have addressed the combination therapy of sorafenib/sunitinib and chemotherapeutic reagents, such as platinum, taxane, and gemcitabine (9-14), whereas only a small number of studies have evaluated the combination with fluorinated pyrimidine $(11,22)$. The present findings are consistent with the result reported by Takeuchi et al (22), who examined the combination effect of S-1 (an oral fluorinated pyrimidine) and sorafenib for RCC treatment in vitro and in tumor-bearing murine models. Synergistic anti-proliferative effects were observed in vitro and in vivo, and the authors revealed that suppression of TS and E2F-1 expression contributed to the synergism. A phase I study, including 73 patients with advanced solid tumors, assessed the tolerability and effect of sunitinib in combination with capecitabine, which is an oral fluorinated pyrimidine (11). There were no clinically significant pharmacokinetic drug-drug interactions, and most adverse events, which were mild to moderate in severity, were manageable with dose reduction and rarely led to study withdrawal. Partial responses were confirmed in 9 (12.3\%) patients. The authors concluded that the combination of sunitinib and capecitabine resulted in an acceptable safety profile in patients with advanced solid tumors. However, further evaluation of sorafenib/sunitinib in combination with fluorinated pyrimidine antitumor reagents may be required.

Sorafenib and sunitinib bind reversibly to the ATP binding site of their target kinases, thereby inhibiting their catalytic activity. The two inhibitors show significant activity against neovascularization-related RTKs, such as VEGFR2, VEGFR3, and PDGFR- $\beta(23,24)$. Although one of the most noteworthy target kinases of sorafenib is Raf- 1 , the contribution of the Raf kinase inhibitory properties of sorafenib to the antitumor effect in RCC is not completely understood (1). In the present study, to identify markers predicting the sensitivity to MKIs, we investigated the mRNA expression levels of major RTKs in RCC cell lines. Our results revealed that PDGFR- $\beta$ was expressed in all the cell lines (Fig. 2). A previous study demonstrated that PDGFR- $\beta$ is found in pericytes that surround capillary endothelial cells, and is involved in stabilizing the vascular endothelium (23). MKIs exert tumor growth inhibitory effects through the suppression of neovascularization, and through direct cytotoxic or cytostatic effects (1). Our findings suggested that the direct cytotoxic or cytostatic effects of sorafenib/sunitinib in RCC cells are mediated by a block in the signaling pathway triggered by PDGFR- $\beta$ phosphorylation. Additionally, our analysis revealed that the higher sensitivity to sunitinib tended to be correlated to a higher PDGFR- $\beta$ expression. These results suggested that PDGFR- $\beta$ expression levels serve as a predictive marker for the efficacy of sunitinib treatment in RCC. More studies, including analyses of the phosphorylation levels of PDGFR- $\beta$ and other protein kinases, are required to clarify the mechanism underlying the direct cytotoxic effect of MKIs against RCC cells.

Examination of the sensitivity of the three RCC cell lines against 5FU and the two MKIs revealed that Caki-2 showed a higher resistance to $5 \mathrm{FU}$ and sorafenib compared to the other two cell lines, which was not the case with sunitinib (Table II). The multidrug-resistant (MDR) phenotype has been widely recognized in cancer chemotherapy. One of the main causes of the MDR phenotype is the overexpression of P-glycoprotein, encoded by the multidrug resistance gene $(M D R l)$ and multidrug resistance-associated proteins (MRP) (23). Findings of a recent study have shown that of the two MKIs, only sorafenib is a substrate for MRP2, a clinically significant member of the MRP transporter family (24). In addition, MRP2 is known to be linked to resistance against 5FU (25). Considering the evidence from previous reports, it is suggested that the overexpression of MRP2 in Caki-2 cells was responsible for the high resistance to both $5 \mathrm{FU}$ and sorafenib.

The major antitumor activity of MKIs is mediated by inhibition of vasculogenesis and angiogenesis, which cannot be reflected in in vitro experiments. Our in vitro experiments revealed the additive effect of combination treatment with $5 \mathrm{FU}$ and MKIs in Caki-1 cells (Fig. 2), whereas in vivo experiments showed that the combination acted synergistically to inhibit the growth of subcutaneous xenograft tumors of Caki-1 cells (Fig. 4A). Assessment of the Ki-67 labeling index and MVD confirmed that the combination therapy decreased cell proliferation as compared to monotherapy (Fig. 4D and E). There is a notable limitation to the present study. The in vivo data were generated only in Caki-1 xenograft tumors. The use of other RCC cell lines is required to accurately assess the clinical potential of the combination of 5FU and MKIs.

In conclusion, we showed that the combination therapy of $5 \mathrm{FU}$ and sorafenib/sunitinib exerts a synergistic anti-tumor effect in human RCC cells. Our findings support the feasibility of the clinical evaluation of combination therapy with fluorinated pyrimidines and MKIs. The regimen should be modified to suit clinical needs, and this protocol may provide an effective and tolerable therapeutic modality for advanced RCC patients.

\section{References}

1. Rini BI: Vascular endothelial growth factor-targeted therapy in renal cell carcinoma: current status and future directions. Clin Cancer Res 13: 1098-1106, 2007.

2. Wagstaff J: Renal cell cancer: is immunotherapy dead? Ann Oncol 18 (Suppl 9): 94-97, 2007.

3. Ferrara N: Vascular endothelial growth factor as a target for anticancer therapy. Oncologist 9 (Suppl 1): 2-10, 2004

4. Wilhelm SM, Carter C, Tang L, et al: BAY 43-9006 exhibits broad spectrum oral antitumor activity and targets the RAF/ MEK/ERK pathway and receptor tyrosine kinases involved in tumor progression and angiogenesis. Cancer Res 64: 7099-7109, 2004.

5. Roskoski R Jr: Sunitinib: a VEGF and PDGF receptor protein kinase and angiogenesis inhibitor. Biochem Biophys Res Commun 356:323-328, 2007.

6. Hutson TE: Targeted therapies for the treatment of metastatic renal cell carcinoma: clinical evidence. Oncologist 16 (Suppl 2): 14-22, 2011.

7. Escudier B, Eisen T, Stadler WM, et al: Sorafenib for treatment of renal cell carcinoma: final efficacy and safety results of the phase III treatment approaches in renal cancer global evaluation trial. J Clin Oncol 27: 3312-3318, 2009.

8. Motzer RJ, Hutson TE, Tomczak P, et al: Overall survival and updated results for sunitinib compared with interferon alfa in patients with metastatic renal cell carcinoma. J Clin Oncol 27: 3584-3590, 2009

9. Dal Lago L, D'Hondt V and Awada A: Selected combination therapy with sorafenib: a review of clinical data and perspectives in advanced solid tumors. Oncologist 13: 845-858, 2008.

10. Ulahannan SV and Brahmer JR: Antiangiogenic agents in combination with chemotherapy in patients with advanced non-small cell lung cancer. Cancer Invest 29: 325-337, 2011.

11. Sweeney CJ, Chiorean EG, Verschraegen CF, Lee FC, Jones S, Royce M, Tye L, Liau KF, Bello A, Chao R and Burris HA: A phase I study of sunitinib plus capecitabine in patients with advanced solid tumors. J Clin Oncol 28: 4513-4520, 2010. 
12. Castillo-Avila W, Piulats JM, Garcia Del Muro X, Vidal A, Condom E, Casanovas O, Mora J, Germà JR, Capellà G, Villanueva A and Viñals F: Sunitinib inhibits tumor growth and synergizes with cisplatin in orthotopic models of cisplatinsensitive and cisplatin-resistant human testicular germ cell tumors. Clin Cancer Res 15: 3384-3395, 2009.

13. Zhang D, Hedlund EM, Lim S, Chen F, Zhang Y, Sun B and Cao Y: Antiangiogenic agents significantly improve survival in tumor-bearing mice by increasing tolerance to chemotherapyinduced toxicity. Proc Natl Acad Sci USA 108: 4117-4122, 2011.

14. Yoon CY, Lee JS, Kim BS, Jeong SJ, Hong SK, Byun SS and Lee SE: Sunitinib malate synergistically potentiates anti-tumor effect of gemcitabine in human bladder cancer cells. Korean J Urol 52: 55-63, 2011.

15. Hartmann JT and Bokemeyer C: Chemotherapy for renal cell carcinoma. Anticancer Res 19: 1541-1543, 1999.

16. Ravaud A, Trufflandier N, Ferrière JM, Debled M, Palussière J, Cany L, Gaston R, Mathoulin-Pélissier S and Bui BN: Subcutaneous interleukin-2, interferon alpha-2b and 5-fluorouracil in metastatic renal cell carcinoma as second-line treatment after failure of previous immunotherapy: a phase II trial. Br J Cancer 89: 2213-2218, 2003.

17. Miyake M, Fujimoto K, Tanaka M, Hirao Y, Uemura H, Otani T and Yoshii M: Immunochemotherapy with interferon- $\alpha$, interleukin-2, 5-fluorouracil, and cimetidine for patients with advanced renal cell carcinoma. J Nara Med Assoc 60: 37-47, 2009.

18. Naito S, Eto M, Shinohara N, Tomita Y, Fujisawa M, Namiki M, Nishikido M, Usami M, Tsukamoto T and Akaza H: Multicenter phase II trial of S-1 in patients with cytokine-refractory metastatic renal cell carcinoma. J Clin Oncol 28: 5022-5029, 2010.
19. Miyake M, Fujimoto K, Anai S, Ohnishi S, Nakai Y, Inoue T, Matsumura Y, Tomioka A, Ikeda T, Okajima E, Tanaka N and Hirao Y: Inhibition of heme oxygenase-1 enhances the cytotoxic effect of gemcitabine in urothelial cancer cells. Anticancer Res 30: 2145-2152, 2010 .

20. Miyake M, Fujimoto K, Anai S, Ohnishi S, Kuwada M, Nakai Y, Inoue T, Matsumura Y, Tomioka A, Ikeda T, Tanaka N and Hirao Y: Heme oxygenase-1 promotes angiogenesis in urothelial carcinoma of the urinary bladder. Oncol Rep 25: 653-660, 2011.

21. Koch I, Depenbrock H, Danhauser-Riedl S, Rastetter JW and Hanauske AR: Interleukin 1 modulates growth of human renal carcinoma cells in vitro. Br J Cancer 71: 794-800, 1995.

22. Takeuchi A, Shiota M, Tatsugami K, Yokomizo A, Eto M, Inokuchi J, Kuroiwa K, Kiyoshima K and Naito S: Sorafenib augments cytotoxic effect of S-1 in vitro and in vivo through TS suppression. Cancer Chemother Pharmacol 68: 1557-1564, 2011.

23. Naito S, Yokomizo A and Koga H: Mechanisms of drug resistance in chemotherapy for urogenital carcinoma. Int J Urol 6: 427-439, 1999.

24. Shibayama Y, Nakano K, Maeda H, Taguchi M, Ikeda R, Sugawara M, Iseki K, Takeda Y and Yamada K: Multidrug resistance protein 2 implicates anticancer drug-resistance to sorafenib. Biol Pharm Bull 34: 433-435, 2011.

25. Wang EJ and Johnson WW: The farnesyl protein transferase inhibitor lonafarnib (SCH66336) is an inhibitor of multidrug resistance proteins 1 and 2. Chemotherapy 49: 303-308, 2003. 